\title{
Web Shopping Support System for Elderly People using WebRTC
}

\author{
Naoya HONGO, Hiroshi YAMAMOTO, Katsuyuki YAMAZAKI \\ Nagaoka University of Technology, 1603-1 Kamitomioka, Nagaoka, Niigata 940-2188 Japan \\ Contact: naohon@stn.nagaokaut.ac.jp
}

\begin{abstract}
An increase in elderly shopping refugees is considered as a serious social problem. Therefore, this paper proposes a new web shopping support system for the elderly shopping refugees. Features of the proposed system are 1) to use WebRTC for synchronizing displayed web pages instead of a centralized proxy server, 2) setup procedures of end-to-end communication through NAT devices by utilizing a general mail service, 3) a mutual authentication method using video streaming of the WebRTC technology, and 4) remote assistance methods for teaching shopping refugees how to use EC sites. Evaluation of the proposed methods has shown that the WebRTC technology and the proposed remote assistance methods are effective for the web shopping support.
\end{abstract}

Keywords - Browsers synchronization, WebRTC, Shopping refugee, NAT traversal, HTML5

\section{INTRODUCTION}

It is difficult for elderly shopping refugees living in hilly and mountainous areas to go shopping [1]. An increase in these shopping refugees is considered as a social problem. In addition, with a spread of useful electronic commerce (EC) sites, many people can buy necessary supplies at home. Furthermore, the Internet can be leveraged by not only a desktop/laptop computer but a smartphone/tablet which is equipped with an intuitive and useful interface. However, it is difficult for a lot of elderly people who have not used the Internet to benefit from these technologies [2]. Therefore, a remote assistance system for the elderly people is attracting attention [3]-[6]. However, the existing remote assistance systems utilize a centralized proxy server to synchronize displayed contents on user terminals through NAT devices or cannot execute NAT traversal.

On the other hand, with the revolutionary advances in web browsers, a real-time peer-to-peer communication is achieved by a new WebRTC technology [7], [8]. The WebRTC technology can easily establish sessions of video and audio streams between web browsers through NAT devices. Furthermore, in this year, the WebRTC technology has supported Data Channel APIs so as to transmit binary and text data. The Data Channel can achieve secure and reliable communication between end-devices.

Therefore, this paper proposes a new web shopping support system for the elderly shopping refugees by using the WebRTC technology. The proposed system provides synchronizing displayed web pages between user terminals. Furthermore, the system is equipped with an assistance function which helps a supporting person to teach a shopping refugee how to use EC sites from a remote place.

\section{RELATED WORKS AND OBJECTIVES OF THIS STUDY}

\section{A. Problem of Shopping Refugees and IT Shortfalls}

Japanese Ministry of Economy, Trade and Industry, announced that 6 million people of shopping refugees exist and most of them are elderly people [1]. The shopping refugees live in severe places such as hilly and mountainous areas, hence it is difficult for them to go shopping. For example, a heavy snowfall prevents them from walking outside in the winter. Furthermore, there are many inconvenient regions where there are few shops. Problems of the shopping refugees become an important issue in sociologies, and hot study topics by many researches [9], [10].

On the other hand, people can buy necessary supplies at home by using EC sites. Some local supermarkets, major supermarket chains and companies of a convenience-store have started the EC sites in Japan. Nevertheless, it is difficult for IT shortfalls such as elderly people to benefit from the technology [2]. In fact, the utilization of the Internet in the elderly people is very low (about $30 \%$ ). In the future, such a digital divide will also be a serious problem in other countries.

Therefore, in order to start utilizing the convenient services, the elderly person should be taught how to use the on-line services. Since the elderly shopping refugees might not reside close to the supporting person, a remote assistance system is necessary.

\section{B. Existing Studies for Shopping Refugees and IT Shortfalls}

Existing remote assistance systems and studies [3], [4], [5] support the IT shortfalls by synchronizing contents displayed on a web browser between an IT shortfall and a supporting person. However, these systems have need of a centralized proxy server for the synchronization. Management of the server requires a lot of cost and the centralized system cannot achieve scalability.

Furthermore, Remote Framebuffer Protocol [11] used by Virtual Network Computing (VNC) tools has been developed. The VNC tools are used to control a remote computer through the Internet. However, the VNC tools cannot control a remote smartphone/tablet because a user application cannot normally obtain full privilege of the terminal. Furthermore, the data 
traffic cannot automatically traverse NAT devices (i.e., home router) in these systems. Even if a NAT traversal method is implemented in these systems, a new server is necessary to traverse the NAT devices.

In addition, a synchronization system using WebSocket technology is studied [6], [12], which synchronizes contents between web browsers. This system is achieved by developing the add-on of a web browser. However, this system cannot work through the NAT devices.

Finally, in these existing systems and a related study [13], a communication method (e.g., voice chat) is not considered, and an assistance method is also not implemented for user to learn how to utilize web browsing.

\section{WebRTC as Communications}

In recent years, a real-time peer-to-peer communication technology, WebRTC, has been developed and studied [14]. Especially, the browser APIs and the relevant protocols of the WebRTC technology are standardized in the World Wide Web Consortium (W3C) and the Internet Engineering Task Force (IETF). Users can communicate through video and audio session established by the WebRTC technology.

The WebRTC technology achieves NAT traversal by using the Trickle ICE technology [15]. The ICE provides a framework to establish WebRTC connections by automatically selecting an appropriate NAT traversal method.

Due to the recent update, the WebRTC technology can also transmit binary and text data via Data Channel APIs. The Data Channel is established by Stream Control Transmission Protocol (SCTP) over Datagram Transport Layer Security (DTLS). The Data Channel provides a secure connection using the DTLS and a reliable communication applying the SCTP. Therefore, applications such as sharing files can be developed easily by using the Data Channel on the web browsers.

\section{Objectives of this Study}

In this study, in order to support the shopping refugees, we propose a web shopping support system that does not use the centralized proxy server, and that works even if user terminals are placed behind the NAT devices. Furthermore, the proposed system also provides remote assistance methods to support shopping on the EC sites. In addition, this system utilizes the WebRTC technology to synchronize contents and to communicate between users. Therefore, the proposed system can be used by simply installing a web browser into a user terminal.

\section{III.PROPOSED SYSTEM}

\section{A. Overview of Proposed System}

Figure 1 indicates an overview of the proposed web shopping support system. This proposed system provides mutual communication between user terminals without preparing any server. In order to directly exchange synchronization information (e.g., the URL of a web site) between user terminals, the system should traverse NAT devices because a home network tends to be constructed inside a NAT router.

This proposed system is assumed to be used by both a shopping refugee and a supporting person such as the family. The shopping refugee uses an Android tablet in this system because the device is equipped with a touch-screen as a userfriendly interface. Two procedures are required before starting synchronization of displayed contents between proposed web browsers. First, this system traverses the NAT devices by leveraging a Session Traversal Utilities for NAT [16] (STUN) server and a general mail service. Second, the users authenticate the counterparts using face-to-face communication. Finally, the system exchanges synchronization information between the proposed web browsers.

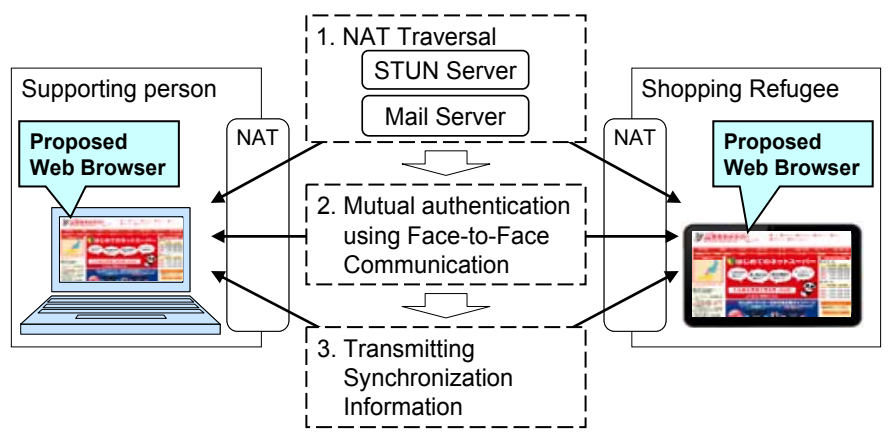

Figure 1. Overview of the proposed system

\section{B. Proposed Method using WebRTC}

In our proposed system, WebRTC technology is used for communication between user terminals. Figure 2 shows a functional structure of the proposed system using the WebRTC technology. Two components are required within a terminal, 1) the proposed Synchronization Web Browser (SWBrowser), and 2) a WebRTC application running over general web browsers such as Google Chrome.

A web browser layer in the SWBrowser leverages a WebView component in Java FX or Android. The WebView component provides an ability of displaying web pages. The web pages can easily browse and append a new graphic for sharing the browsing position by the WebView component. However, the WebRTC technology is not contained within the current WebView component although the general web browsers implement the technology. For these reasons, the structure of our proposed system is divided into the SWBrowser executed on Java virtual machine and the WebRTC application run on the general web browsers.

The SWBrowser consists of three functional layers. The web browser layer transmits the URL of a web site or an identifier of a HTML component to a gatekeeper layer in the SWBrowser when a displayed content on the SWBrowser is changed. The web browser layer also notifies the gatekeeper layer of data for a remote assistance method, which will be explained later. If a web page cannot be shared (e.g., a purchase screen in EC sites), the gatekeeper layer requests creating the screen-shot of the displayed web contents to the 
own web browser layer, and the obtained screen-shot is sent to the gatekeeper layer as an event.

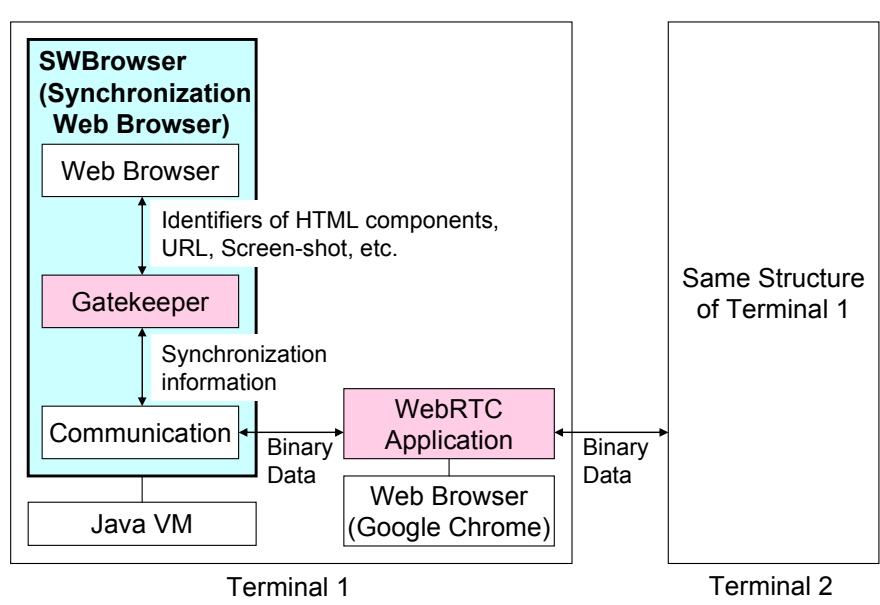

Figure 2. Structure of our proposed system using WebRTC

Next, the gatekeeper layer transmits the synchronization information to a communication layer in the SWBrowser. The information consists of data notified from the web browser layer and an attribute code of the data. In addition, the gatekeeper layer blocks the synchronization until a mutual authentication of face-to-face communication succeeds.

Finally, the communication layer connects to the WebRTC application via a WebSocket technology on the Google Chrome as the general web browser. This layer runs a web server and a WebSocket server for exchanging binary data with the WebRTC application. The WebRTC application transmits the synchronization information as binary data to the counterpart through Data Channel APIs of the WebRTC technology.

\section{Setup Procedures using Public Servers}

Figure 3 indicates setup procedures of NAT traversal between user terminals. The setup of the connection through the WebRTC technology is achieved by exchanging connection information via mail servers. This method assumes a condition where a shopping refugee runs the SWBrowser and is polling the mail server to receive a mail including an offer from a supporting person. First, the supporting person starts the SWBrowser. The general web browser (Google Chrome) is executed by the SWBrowser and accesses the local web server on the SWBrowser. The SWBrowser returns a WebRTC application, and the Google Chrome runs the obtained application. After that, the application checks a global IP address and a port number of own terminal by accessing a STUN server, and creating a Session Description Protocol (SDP) message (e.g., offer/answer for establishing a multimedia session). The identifier and the SDP message are transferred to the SWBrowser through a WebSocket connection. The SWBrowser transmits a mail including them to the shopping refugee. The SWBrowser in the shopping refugee side receives the mail, and executes the same procedures. After that, the WebRTC connection can be established between the proposed systems.

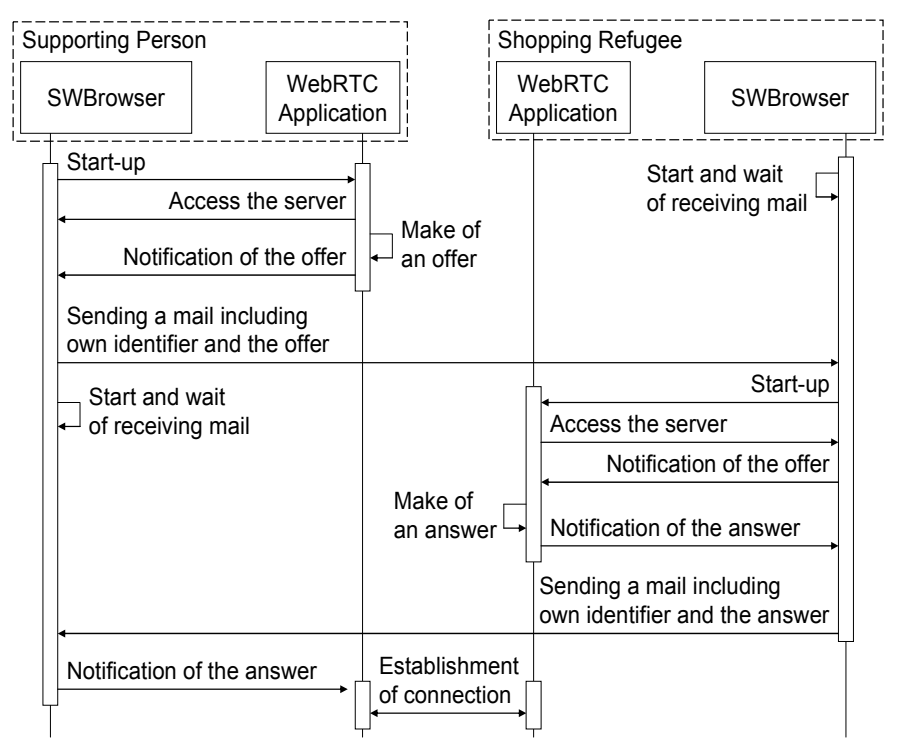

Figure 3. Setup procedures

\section{Mutual Authentication Method using Face-to-Face Communication}

Authentication is necessary for avoiding an error and abuse of our proposed system. Figure 4 shows a flow chart of the proposed mutual authentication method. The proposed method utilizes face-to-face communication through the video streaming of the WebRTC technology. After the WebRTC connection is established, the Google Chrome displays a mutual authentication screen so that the users can decide to accept synchronization of the counterpart. When both users accept synchronization, the mutual authentication succeeds.

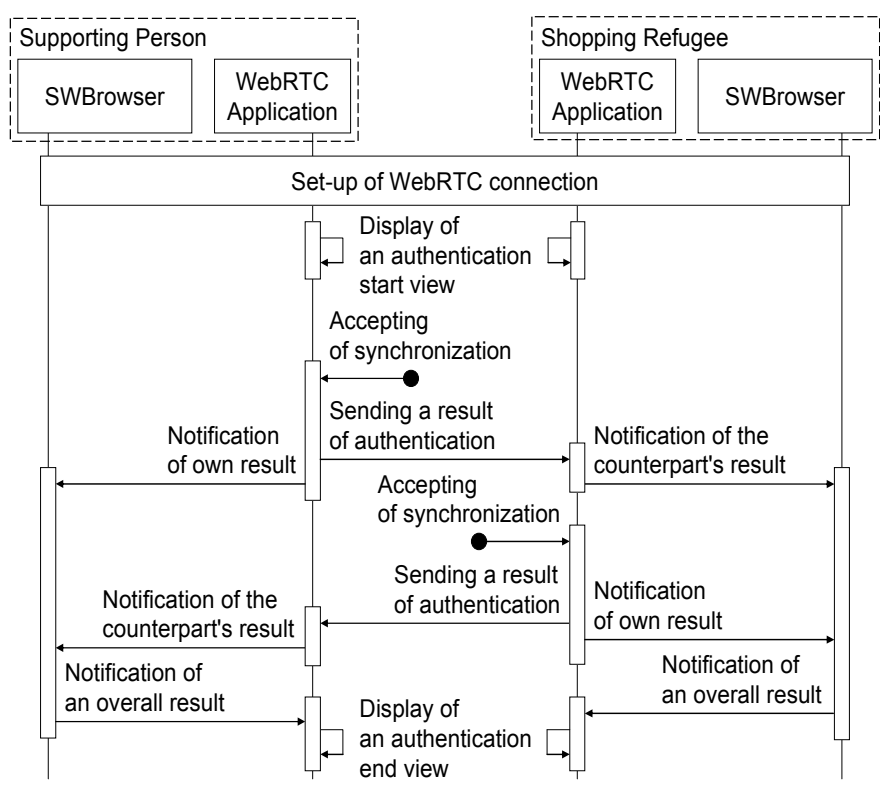

Figure 4. Mutual authentication procedures 


\section{E. Remote Assistance Methods for Web Shopping}

If a mutual communication provides only a voice chat, it is difficult for a shopping refugee to understand where a supporting person points on a web page. Therefore, we propose two remote assistance methods for communication in web shopping.

Figure 5 shows an input assistance method to focus on a text box in the web page. When the supporting person clicks the text box, a same component is focused and a virtual keyboard is displayed on the terminal of the shopping refugee.

Figure 6 illustrates a highlight indication method. This method is provided to notify the shopping refugee of a location where she/he should focus. When the support person clicks some position on the web page, a graphic (e.g., circle) is illustrated on a same position. This method can also work on the screen-shot of the web page that is transmitted from the counterpart.

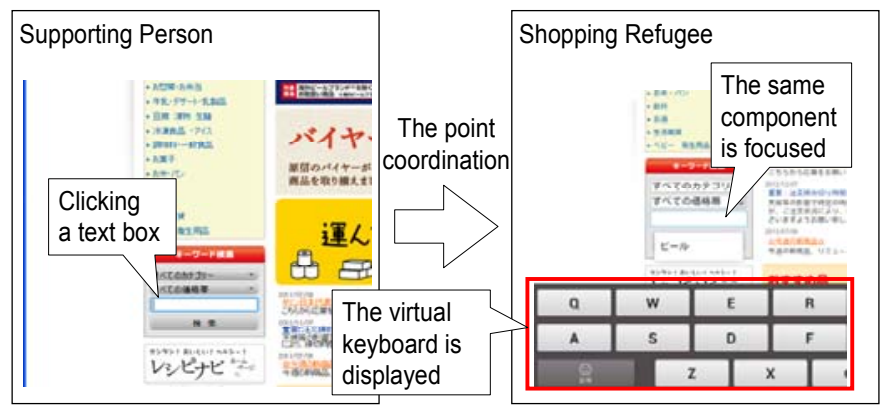

Figure 5. Input assistance method

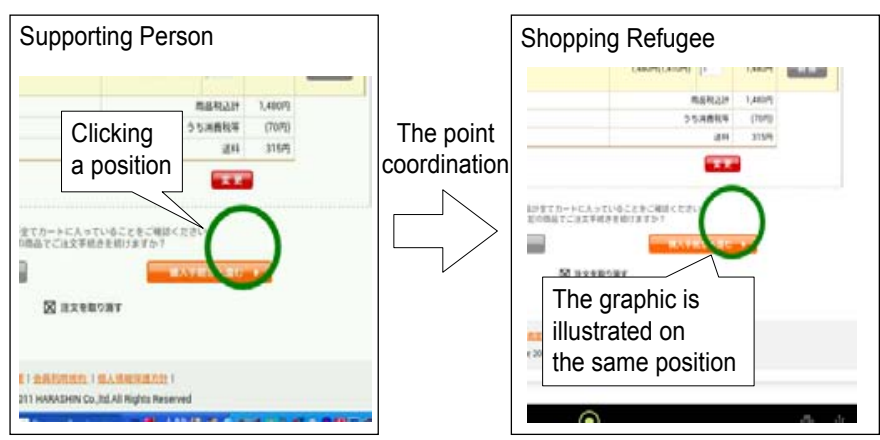

Figure 6. Highlight indication method

\section{Evaluation of Proposed System}

We have implemented the proposed system and evaluated effectiveness of each proposed method.

\section{A. Setup Time in Communication}

We first measured a processing time of the setup procedures of a WebRTC connection between two terminals. Figure 7 shows a measurement environment. The experiments were performed in two conditions where a shopping refugee uses a terminal connected to 1) a $3 \mathrm{G}$ cellular network or 2) a broadband network. A terminal of supporting person is always connected to the broadband network. In each environment, the setup procedures were executed and the processing time was measured thirty times. Here, we used the STUN server provided by Google, the mail server provided by Yahoo! JAPAN, and Google Chrome Beta (ver. 31) as a web browser running the WebRTC.

Figure 8 indicates the processing time in each step of the setup procedures. When the terminal is connected to the $3 \mathrm{G}$ cellular network, the processing time tends to be large. As shown in Figure 8, the type of access networks mainly affects the time required for transferring a mail from the terminal of the shopping refugee side to the counterpart. However, the overall processing time does not exceed 13 seconds. On the other hand, when both terminals use the broadband network, the overall processing time can be kept within 9 seconds.

Table 1 summarizes the processing time required for the overall time of each condition. A standard deviation is 2.0 seconds in the case where the shopping refugee used the $3 \mathrm{G}$ cellular network, hence the setup using the $3 \mathrm{G}$ cellular network is completed within 14 seconds with high probability. On the other hand, a standard deviation is 1.1 seconds in the case where both of them are connected to the broadband network. Consequently, the processing time can be always kept within 10 seconds over the broadband network.

In case of the $3 \mathrm{G}$ cellular network, the delay is caused by a slow RTT and a narrow bandwidth, and a bottleneck of the proposed method is a communication with the mail server. However, it is concluded that the proposed NAT traversal method achieves the sufficient performance for the browser synchronization because the process can be completed within 14 seconds even when the $3 \mathrm{G}$ cellular network is adopted.

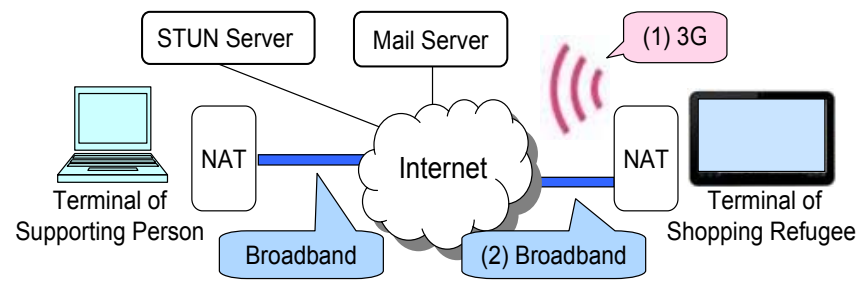

Figure 7. Measurement environment of setup time in communication

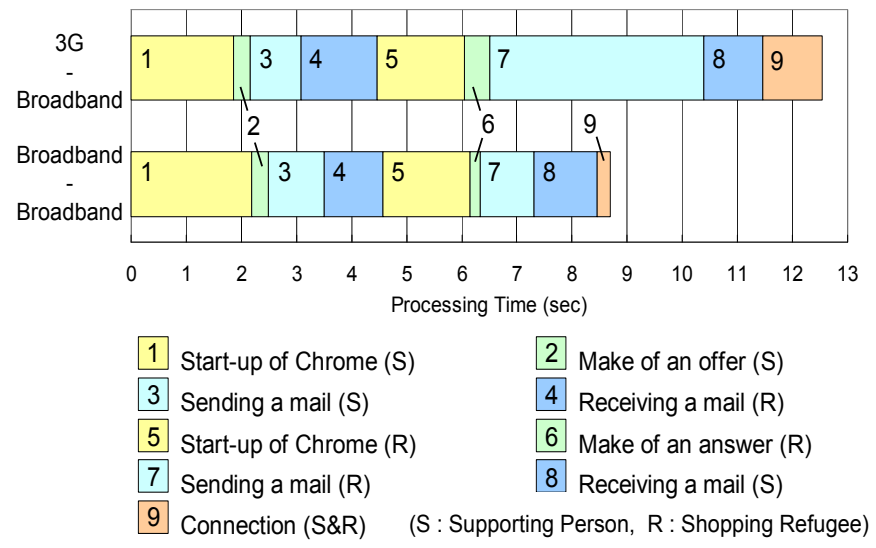

Figure 8. Setup time in communication 
TABLE 1. Overall Time of Setup

\begin{tabular}{|c|c|c|}
\hline & $\begin{array}{c}\text { 3G - } \\
\text { Broadband }\end{array}$ & $\begin{array}{c}\text { Broadband - } \\
\text { Broadband }\end{array}$ \\
\hline Average Processing Time [sec] & 12 & 8.7 \\
\hline Standard Deviation [sec] & 2.0 & 1.1 \\
\hline
\end{tabular}

\section{B. Implementation of Mutual Authentication Method}

The mutual authentication method is implemented in the proposed system. Figure 9 shows screens of mutual authentication. The users communicate between each other by using the WebRTC application on the Google Chrome. Until both users press a "Yes" button, the synchronization cannot be started by the gatekeeper layers in the SWBrowsers. When both users accept synchronization, the gatekeeper layers starts to control synchronization between the SWBrowsers.

From the experimental evaluation, it has been concluded that the WebRTC technology appropriately helps a user to confirm the counterpart and to decide whether or not she/he permits to synchronize contents and to communicate between both users.
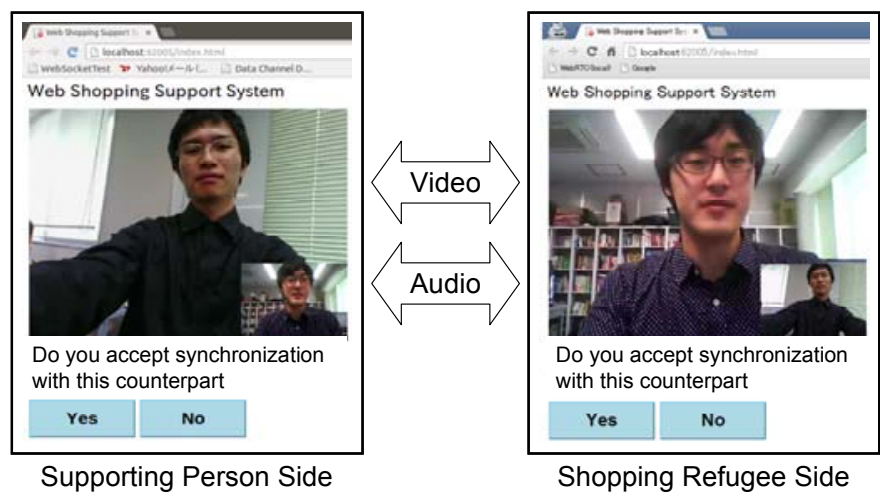

Figure 9. Mutual authentication screens

\section{Effectiveness of Remote Assistance Methods}

We have evaluated effectiveness of the implemented remote assistance methods. In the evaluation, five subjects were selected as the shopping refugees. They are from forties to seventies and have never utilized any EC site.

\section{1) Input Assistance Method}

Figure 10 illustrates virtual keyboards that the input assistance method displays on a user terminal of an Android tablet. Figure 10 (a) is a PC input method (i.e., QWERTY design) and Figure 10 (b) is a cellular phone input method (i.e., design of a cellular phone). In order to decide appropriate method that the subjects fast complete a text input, we measured input time required for the subjects to enter an ID and a password as login information of an EC site by using each virtual keyboard. The ID is a fanciful e-mail address comprised of 16 alphanumeric characters and the password is 8 alphanumeric characters. The subjects input the login information three times in each virtual keyboard.

Figure 11 shows average input time of five persons in each trial. As a result, we have confirmed that the input time of the
PC input method is faster than the cellular phone input method. In addition, training effect has been confirmed in only the PC input method. Therefore, it has been concluded that the virtual keyboard of the PC input method is appropriate for proposed system.

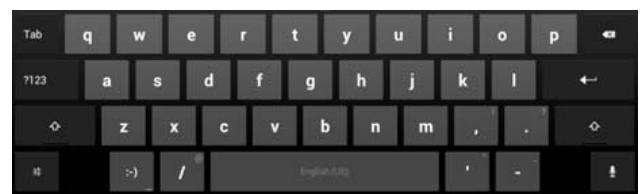

(a) PC input method

\begin{tabular}{|c|c|c|c|c|}
\hline$\pi$ & is & $A B C$ & DEF & $\mathbf{a}$ \\
\hline 4 & GHA & $\mathrm{JKL}$ & MNO & , \\
\hline (9) & POAS & TUV & $w X Y Z$ & 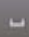 \\
\hline N & $m-A$ & $\because:$ & $i i$ & 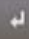 \\
\hline
\end{tabular}

(b) Cellular phone input method

Figure 10. Virtual keyboards displayed in the input assistance method

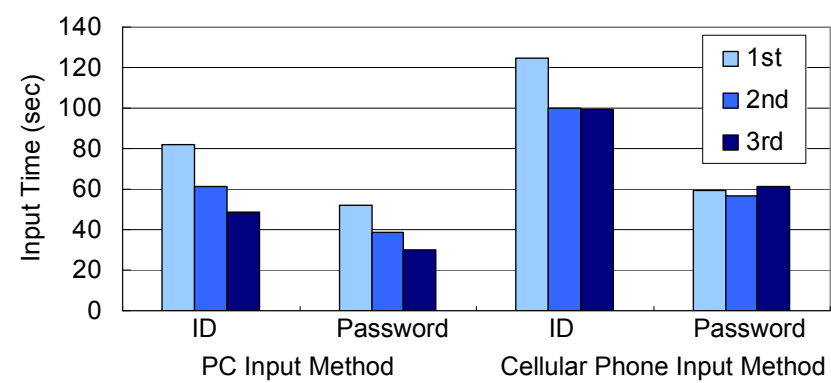

Figure 11. Input time of indicated items

\section{2) Highlight Indication Method}

Figure 12 illustrates forms displayed in the highlight indication method. Figure 12 (a) is a circle form and Figure 12 (b) is an arrow form. In order to examine an appropriate form that the subjects fast complete selection of target items, we measured selection time required for the subjects to click a specified item on an EC site. The subjects selected the item five times when each form is displayed. In addition, the each graphic was also evaluated by asking subjective assessment at points from zero to five.

Figure 13 indicates average selection time of five persons in each method. In the non-supported environment, the selection time was approximately 10 seconds. In contrast, when the highlight indication method was used, the subjects could select the items within 4 seconds. Besides, the average point of the subjective assessment of the circle form is 4.8 points while that of the arrow form is 4.0 points. As a result, we have concluded that the highlight indication method by the circle form is more effective in assisting shopping refugees than the arrow form. 


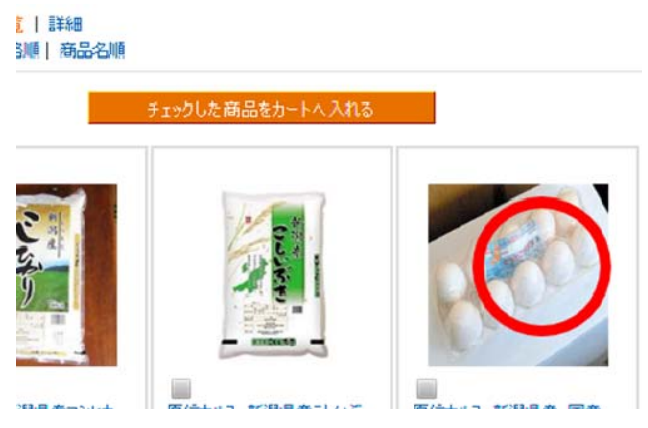

(a) Circle form

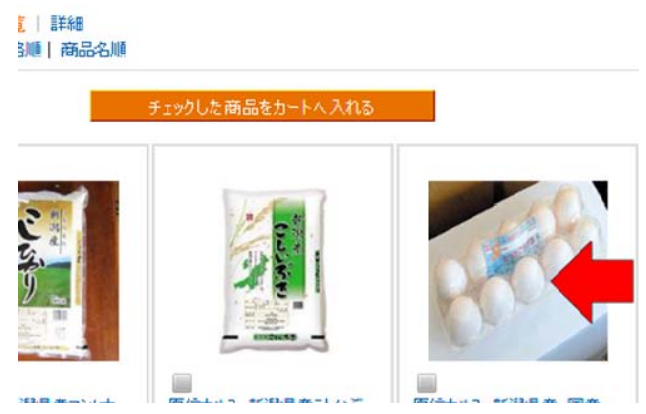

(b) Arrow form

Figure 12. Forms displayed in the highlight indication method

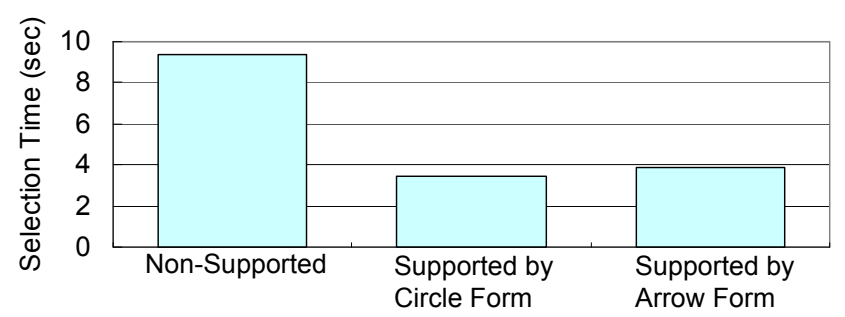

Figure 13. Selection time of indicated items

\section{Conclusions}

This paper has presented a new web shopping support system for elderly shopping refugees. Features of the proposed system are 1) to use a peer-to-peer communication technology, WebRTC, instead of a centralized proxy server, 2) setup procedures of end-to-end communication through NAT devices by utilizing a mail server, 3) to provide a mutual authentication method using video streaming of the WebRTC technology, and 4) two remote assistance methods for teaching shopping refugees how to use EC sites.

Experimental evaluation of the proposed system has shown effectiveness of assisting a web shopping. First, measurement of setup time has indicated that the WebRTC communication can start within 13 seconds even when the $3 \mathrm{G}$ cellular network is adopted. As a result, the WebRTC connection can be established within sufficiently short interval. Next, the mutual authentication method is implemented by using a video and audio streaming function of the WebRTC APIs. From these results, it has been concluded that the WebRTC technology is efficient for building the proposed system. Finally, the virtual keyboard of a PC input method is appropriated in the proposed system, and a highlight indication method enables shopping refugees to select a target item on an EC site within 4 seconds.

In the future study, we will develop a content translation function which translates displayed contents on a web site to an appropriate form for elderly shopping refugees. Furthermore, our system will run on the proposed SWBrowser only when the WebRTC is implemented in WebView components. In addition, the system will be extended so that one-to-many synchronization can be supported in EC sites.

This study was partly supported by the KDDI Foundation, Japan.

\section{REFERENCES}

[1] Japan Ministry of Economy, Trade and Industry, "Report by the Study Group on the Role of Distribution Systems in Community Infrastructure," May 2010.

[2] Japan Ministry of Internal Affairs and Communications, "2012 WHITE PAPER Information and Communications in Japan," July 2013.

[3] D. Nakamura, and T. Hikita, "Collaborative Web Browsing System Realizing Sharing of Practical Page Operations", Forum on Information Technology Proc. Fourth fascicle, pp.467-472, August 2009.

[4] N. Imai, M. Isomura, and K. Yoshihara, "A Discussion on Synchronized Web Browsing for Group Communications," Communications Society Conference of The Institute of Electronics, Information and Communication Engineers Proc. Communication 2, pp.98, September 2010.

[5] Q. Shen, N. Nakaya, and Y. Koi, "Supporting the aged people by using Remote Support System," Communications Society Conference of The Institute of Electronics, Information and Communication Engineers Proc. Communication 2, pp.297, September 2008.

[6] T. Yogi, T. Shiroma, T. Nagata, Y.Taniguchi, and S. Tamaki, "Study on P2P Communication between Web Browsers using WebSocket," The Institute of Electronics, Information and Communication Engineers Technical Report NS, Vol.110 No.372, pp.59-62, January 2011

[7] WebRTC, http://www.webrtc.org/, October 2013.

[8] T. Hishiki, T. Ohba, and A. Koike, "Standardization Trends of WebRTC Technology," The Institute of Electronics, Information and Communication Engineers Journal, Vol.96 No.10, pp.790-796, October 2013.

[9] A. Taketa, Y. Komatsu, and I. Yokomizo, "Development of Support System by Cooperatives for People at a Disadvantage in Shopping in Hi11y and Mountainous Areas," Journal of Rural Problems, Vol.48 No.1, pp.145-150, June 2012.

[10] Y. Takahashi, Y. Takeda, and S. Ouchi, "Business Models and Governmental Assistance for Mobile Shops: A Case of Shoei Keiran, Ikoma City, Nara Prefecture," Shokei-gakuso: Journal of Business Studies, Vol.58 No.3, March 2012.

[11] T. Richardson, "The RFB Protocol," http://www.realvnc.com/, November 2010.

[12] WebSocket, http://www.websocket.org/, October 2013.

[13] N. Hongo, H. Yamamoto, K. Yamazaki, "Browser Synchronization System for Supporting Elderly People and IT Shortfalls," Proc. IEEE Computers, Software \& Applications Conference 2013, pp.455-458, July 2013.

[14] C. Vogt, M. J. Werner, T. C. Schmidt, "Leveraging WebRTC for P2P Content Distribution in Web Browsers," 21st IEEE International Conference on Network Protocols 2013 Demo Session, October 2013.

[15] E. Ivov, J. Uberti, and E. Ivov, "Trickle ICE: Incremental Provisioning of Candidates for the Interactive Connectivity Establishment (ICE) Protocol," IETF Internet Draft, draft-ivov-mmusic-trickle-ice-01, March 2013.

[16] J. Rosenberg, R. Mahy, P. Matthews, and D. Wing, "Session Traversal Utilities for NAT (STUN)," RFC 5389, October 2008.

[17] M. Handley, V. Jacobson, and C. Perkins, "SDP: Session Description Protocol,", RFC 4566, June 2006. 


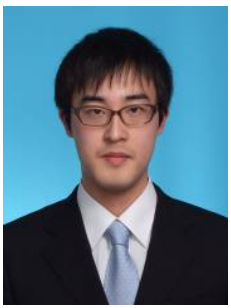

Naoya Hongo received B.E. degree from Nagaoka University of Technology in '12. He is currently a graduate school student in Nagaoka University of Technology. His research interests include computer networks and networked services.

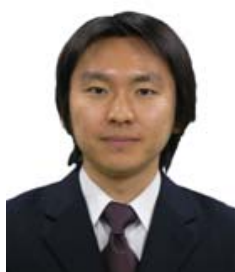

Hiroshi Yamamoto received M.E. and D.E. degrees from Kyushu Institute of Technology, Iizuka, Japan in '03 and '06, respectively. From April '06 to March '10, he worked at FUJITSU LABORATORIES LTD., Kawasaki, Japan. Since April '10, he has been an Assistant Professor in the Department of Electrical

Engineering, Nagaoka University of Technology. His research interests include computer networks, distributed applications, and networked services. He is a member of the IEEE.

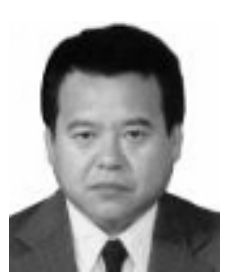

Technology.

Katsuyuki Yamazaki received B.E. and D.E degrees from the University of Electro-communications and Kyushu Institute of Technology in '80 and '01, respectively. At KDD Co. Ltd., he had been engaged in R\&D and international standardization of ISDN, S.S No.7, ATM networks, L2 networks, IP networks, mobile and ubiquitous networks, etc., and was responsible for R\&D strategy of KDDI R\&D Labs. He is currently a Professor of Nagaoka University of 